\title{
Generation of electrical energy at gas pipelines using a transported natural gas technological pressure drop
}

\author{
M.D. Buranov ${ }^{1}$, A.A. Mukolyants ${ }^{1, *}$, I.V. Sotnikova ${ }^{1, * *}$ \\ ${ }^{1}$ Tashkent State Technical University, Tashkent, Uzbekistan
}

\begin{abstract}
The article discusses the possibilities of generating electricity without burning fuel by expanding high-pressure natural gas at gas distribution stations with lower specific capital costs. It is proposed to reduce the pressure of the transported natural gas using expander-generator units instead of traditional throttle devices.
\end{abstract}

Currently, renewable forms of energy are one of the promising areas of scientific and technological progress. Renewable types of energy include solar, wind, heat, tidal energy, as well as the development of principles for the return of part of the energy spent on transporting natural and associated petroleum gases through main pipelines from source to consumer.

The problem of energy conservation, being one of the most important problems in all developed countries, is becoming especially acute in Uzbekistan.

With the growth of the economy and the standard of living of the population, the demand for energy has also increased. In this regard, in recent times, the generating capacities of the country's power system have noticeably increased. A $300 \mathrm{MW}$ power unit was commissioned at New Angren Thermal Power Plant (TPP), as well as an 800 MW unit at Talimarjan TPP; a project was completed to expand Navoi TPP with the construction of a combined cycle gas turbine unit (CCGT) with a capacity of $478 \mathrm{MW}$, and a cogeneration gas turbine unit was introduced at the Tashkent Thermal Power Plant. with a capacity of $27 \mathrm{MW}$. In the framework of projects on the use of alternative and renewable energy sources, expansion units were introduced at Syrdarya and Talimarjan TPPs. At the beginning of 2019, Epsilon Development Company commenced re-commissioning of Khujum well No. 2 (Kultak-Kamash investment block) and the overhaul was initiated, the work on perforation in the range of 3660-3718 meters was performed. After acid treatment of the well, an industrial influx of natural gas in the amount of about 145-180 thousand cubic meters per day was obtained.

From the discovered Khujum field, along the newly built $5.1 \mathrm{~km}$ gas pipeline connecting the Khujum field with the gas collection point of Nazarkuduk gas pipeline, gas production was started at the headquarters of Shurtanneftegaz LLC. The company also received an

\footnotetext{
*Corresponding author: arsen5675@mail.ru

*** Corresponding author: word2605@yahoo.com
}

industrial flow of gas from two wells in Talimarjan field. Thus, it is expected that the total volume of hydrocarbons produced by the company will be 1-1.25 million cubic meters per day.

Determining the prospects for improving the gas transmission system of the republic, Uztransgaz joint stock company, in terms of the technical properties of transit transportation, can operate in stages using various options for modernizing production and improving technological processes [1].

Maintaining a high level of energy intensity of the national economy can lead to the fact that unmet demand will make up a significant part of the current energy consumption in the republic. It will be impossible to cover this demand, given the deterioration of the fixed assets of the existing energy system, its high capital intensity and inertia, without intensifying work in the field of energy conservation.

One of the areas of energy conservation is the use of expander-generator units (EGU) for the production of environmentally friendly electricity (without burning fuel) through the use of a technological pressure drop of gas in the systems of transportation via main pipelines and distribution of natural gas. With the gas supply system existing in the republic, the pressure of the transported natural gas is usually reduced in two stages at gas distribution stations (GDS) and gas control points (GCP) and is performed by throttling.

Currently, projects to use excess energy of gas pressure during its reduction in gas distribution and consumption systems in a number of EU countries are aimed at the production of electric energy. However, to date, no practical measures have been taken for the large-scale and effective practical use of this technology in the Central Asian republics, including Uzbekistan. In this regard, for uninterrupted power supply on the linear part of gas pipelines, gas metering devices at gas 
distribution stations and gas storage facilities, and other gas supply facilities, the authors consider it appropriate to use EGU to generate clean electricity by utilizing compressed natural gas energy.
Studies conducted at Urga, Northern Shurtan and Northern Berdak fields (tab. 1,2,3) showed that the use of transported reservoir gas in turboexpander units requires additional processing and bringing it to the relevant technical standards.

Table 1 Initial composition of the reservoir gas of Urga field (\% mol.)

\begin{tabular}{|c|c|c|c|c|c|c|}
\hline \multirow{3}{*}{ Gas components } & \multicolumn{6}{|c|}{ Horizons } \\
\hline & \multicolumn{2}{|c|}{$\mathrm{J}_{3}{ }^{2}$} & \multirow{2}{*}{$\frac{\mathrm{J}_{3}{ }^{3}}{\text { Well } 4}$} & \multirow{2}{*}{$\frac{\mathrm{J}_{3}{ }^{5+6}}{\text { Well } 11}$} & \multicolumn{2}{|c|}{$\mathrm{J}_{3}{ }^{7}$} \\
\hline & Well 7 & Well 8 & & & Well 6 & Well 8 \\
\hline $\mathrm{CH}_{4}$ & 86,75 & 87,40 & 88,30 & 92,54 & 89,18 & 88,41 \\
\hline $\mathrm{C}_{2} \mathrm{H}_{6}$ & 7,05 & 6,60 & 5,57 & 4,09 & 5,98 & 6,52 \\
\hline $\mathrm{C}_{3} \mathrm{H}_{8}$ & 1,85 & 1,85 & 1,98 & 1,01 & 1,54 & 1,81 \\
\hline $\mathrm{iC}_{4} \mathrm{H}_{10}$ & 0,24 & 0,25 & 0,34 & 0,16 & 0.26 & 0,31 \\
\hline $\mathrm{nC}_{4} \mathrm{H}_{10}$ & 0,38 & 0,35 & 0,48 & 0,19 & 0.26 & 0.38 \\
\hline $\mathrm{C}_{5} \mathrm{H}_{12 \text { +higher }}$ & 0,47 & 0,45 & 0,57 & 0,42 & 0,37 & 0,63 \\
\hline $\mathrm{N}_{2+\text { rare }}$ & 3,12 & 2,30 & 2,49 & 0,70 & 0,74 & 1.28 \\
\hline $\mathrm{CO}_{2}$ & 0,14 & 0,80 & 0,27 & 0,89 & 0,67 & 0,66 \\
\hline $\mathrm{H}_{2} \mathrm{~S}$ & - & - & - & - & - & - \\
\hline $\begin{array}{l}\text { Content of } \mathrm{C}_{5 \text { +higher: }} \text { : } \\
-\% \mathrm{~mol} \text {. } \\
-\mathrm{g} / \mathrm{m}^{3}\end{array}$ & $\begin{array}{l}0.47 \\
22,3 \\
\end{array}$ & $\begin{array}{l}0,45 \\
22,3 \\
\end{array}$ & $\begin{array}{l}0,57 \\
22,3 \\
\end{array}$ & $0,42 \quad 22,3$ & $\begin{array}{l}0,37 \\
22,3 \\
\end{array}$ & $\begin{array}{c}0,63 \\
22,3 \\
\end{array}$ \\
\hline Molecular mass of $\mathrm{C}_{5+\text { thigher }}$ & 114,062 & 119,132 & 94,051 & 94,051 & 94,051 & 85,094 \\
\hline Gas density $\left(760 \mathrm{~mm} \mathrm{Hg} 20^{\circ} \mathrm{C}\right), \mathrm{kg} / \mathrm{m}^{3}$ & 0,777 & 0,778 & 0,771 & 0,742 & 0,772 & 0,771 \\
\hline Relative gas density $\left(760 \mathrm{~mm} \mathrm{Hg} 20^{\circ} \mathrm{C}\right)$ & 0,645 & 0,646 & 0,640 & 0,613 & 0,641 & 0,640 \\
\hline
\end{tabular}

Analysis of the obtained data suggests the need to install an additional filter before entering the expander unit for removing mechanical impurities from the gas during transportation and for reducing the percentage of condensate.

A characteristic feature of the turbo-expander design developed by the authors is location of the turbine and compressor wheels on one shaft, which determines the predominant use of radial or radial-axial runners in order to simplify the design of the supply and removal of the working medium.

In this regard, to use the physical energy of the gas, obtained by reducing the pressure on GDS and GCP, instead of traditional throttle devices, it is advisable to use EGU, which allow to generate electricity due to the pressure drop of the gas [2-5].

Table 2. Initial composition of the reservoir gas of ShimoliyBerdak gas condensate field

\begin{tabular}{|c|c|c|c|c|c|c|c|c|c|}
\hline \multirow[b]{3}{*}{$\begin{array}{l}\text { Composition of the } \\
\text { gas (molar \%) }\end{array}$} & \multirow{3}{*}{\begin{tabular}{|l}
$\mid$ Res. $_{3}{ }^{11}$ \\
well 9 \\
$\begin{array}{l}\text { Perforation } \\
\text { range } \\
1577-1566, \\
(\mathrm{~m})\end{array}$ \\
\end{tabular}} & \multicolumn{4}{|c|}{ Res. $\mathrm{J}_{3}{ }^{9}$} & \multirow{3}{*}{$\begin{array}{l}\quad \text { Res. } \mathrm{J}_{3}{ }^{8} \\
\text { well } 6 \\
\text { Perforation } \\
\text { range } \\
2226-2217, \\
(\mathrm{~m})\end{array}$} & \multicolumn{3}{|c|}{ Res. $\mathrm{J}_{2}{ }^{7}$} \\
\hline & & well 3 & well 7 & well 14 & \multirow[b]{2}{*}{ 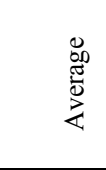 } & & well 7 & well 10 & \multirow[b]{2}{*}{$\begin{array}{l}\mathscr{8} \\
\frac{\pi}{\pi} \\
\frac{\pi}{0} \\
\sum\end{array}$} \\
\hline & & \begin{tabular}{|l|}
$\begin{array}{l}\text { Perforation } \\
\text { range }\end{array}$ \\
$1988-1980$ \\
$(\mathrm{~m})$ \\
\end{tabular} & \begin{tabular}{|l|} 
Perforation \\
range \\
$2024-2017$ \\
$(\mathrm{~m})$
\end{tabular} & \begin{tabular}{|l|}
$\begin{array}{l}\text { Perforation } \\
\text { range }\end{array}$ \\
$1991-1988$, \\
$(\mathrm{m})$
\end{tabular} & & & \begin{tabular}{|l|} 
Perforation \\
range \\
$2222-2218$, \\
$(\mathrm{m})$ \\
\end{tabular} & $\begin{array}{l}\text { Perforation } \\
\text { range } \\
2139-2136, \\
(\mathrm{~m})\end{array}$ & \\
\hline $\mathrm{CH}_{4}$ & 87,12 & 92,21 & 92,31 & 89,95 & 91,49 & 92,95 & 92,90 & 94,08 & 93,49 \\
\hline $\mathrm{C}_{2} \mathrm{H}_{6}$ & 6,36 & 3,81 & 3,05 & 4,0 & 3,62 & 3,78 & 3,10 & 2,41 & 2,76 \\
\hline $\mathrm{C}_{3} \mathrm{H}_{8}$ & 3,05 & 1,53 & 1,50 & 1,72 & 1,58 & 1,31 & 1,48 & 1,23 & 1,36 \\
\hline iso- $\mathrm{C}_{4} \mathrm{H}_{10}$ & 0,78 & 0,24 & 0,20 & 0,50 & 0,31 & 0,22 & 0,16 & 0,32 & 0,24 \\
\hline $\mathrm{n}-\mathrm{C}_{4} \mathrm{H}_{10}$ & 0,82 & 0,23 & 0,17 & 0,42 & 0,27 & 0,22 & 0,15 & 0,30 & 0,23 \\
\hline $\mathrm{C}_{5} \mathrm{H}_{12 \text { thigher }}$ & 1,33 & 0,49 & 0,47 & 1,19 & 0,72 & 0,42 & 0,42 & 0,63 & 0,53 \\
\hline $\mathrm{N}_{2}$ & 0,43 & 1,33 & 1,53 & 1,49 & 1,45 & 0,75 & 1,07 & 0,93 & 1,0 \\
\hline $\mathrm{H}_{2} \mathrm{~S}$ & - & - & - & - & - & - & - & - & - \\
\hline $\mathrm{CO}_{2}$ & 0,11 & 0,16 & 0,77 & 0,73 & 0,55 & 0,35 & 0,72 & 0,10 & 0,41 \\
\hline Total & 100 & 100 & 100 & 100 & 100 & 100 & 100 & 100 & 100 \\
\hline \multicolumn{10}{|c|}{ Potential condensate content, $\mathrm{g} / \mathrm{m}^{3}$} \\
\hline $\begin{array}{l}\text { - in } 1 \mathrm{~m}^{3} \text { of reservoir } \\
\text { gas }\end{array}$ & 57,48 & 23,7 & 22,4 & 51,83 & 32,31 & 20 & 20,3 & 29,32 & 24,81 \\
\hline - in $1 \mathrm{~m}^{3}$ of dry gas & 58,25 & 23,8 & 22,5 & 51,44 & 32,58 & 20,1 & 20,4 & 29,51 & 24,96 \\
\hline $\begin{array}{ll}\begin{array}{l}\text { Potential ethane } \\
\text { content, } \mathrm{g} / \mathrm{m}^{3}\end{array} & \\
\end{array}$ & 79,55 & 47,65 & 38,15 & 50,03 & 45,28 & 47,28 & 38,77 & 30,14 & 34,52 \\
\hline \multicolumn{10}{|l|}{ Critical parameters: } \\
\hline$-\mathrm{P}_{\mathrm{cr} .}, \mathrm{kgf} / \mathrm{cm}^{2}$ & 46,57 & 46,73 & 46,88 & 46,73 & 46,79 & 46,89 & 46,94 & 46,71 & 46,82 \\
\hline$-\mathrm{T}_{\text {cr. }}, \mathrm{K}$ & 211,53 & 199,9 & 199,26 & 199,95 & 199,7 & 199,7 & 199,20 & 198,75 & 198,97 \\
\hline $\begin{array}{l}\text { Gas density at } 20^{\circ} \mathrm{C}, \\
760 \mathrm{~mm} \mathrm{Hg}, \mathrm{kg} / \mathrm{m}^{3}\end{array}$ & 0,824 & 0,746 & 0,746 & 0,746 & 0,746 & 0,739 & 0,741 & 0,739 & 0,740 \\
\hline Relative gas density & 0,684 & 0,62 & 0,620 & 0,619 & 0,619 & 0,613 & 0,615 & 0,613 & 0,614 \\
\hline
\end{tabular}


Continuation of Table 2

\begin{tabular}{|c|c|c|c|c|c|c|c|}
\hline \multirow{3}{*}{$\begin{array}{l}\text { Composition of the } \\
\text { gas (molar \%) }\end{array}$} & \multicolumn{3}{|c|}{ Res. $\mathrm{J}_{2}{ }^{3 \mathrm{a}}$} & \multicolumn{3}{|c|}{ Res. $\mathrm{J}_{2}^{{ }^{2 a}}$} & \multirow{3}{*}{$\begin{array}{c}\text { Res. } \mathrm{J}_{2}{ }^{2 \sigma} \\
\text { well } 16 \\
\begin{array}{c}\text { Perforation range } \\
2615-2605(\mathrm{~m})\end{array}\end{array}$} \\
\hline & well 14 & well 16 & \multirow[b]{2}{*}{ 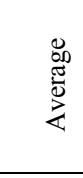 } & well 9 & well 15 & \multirow[b]{2}{*}{ 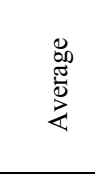 } & \\
\hline & $\begin{array}{l}\text { Perforation range } \\
2468-2463(\mathrm{~m})\end{array}$ & $\begin{array}{l}\text { Perforation range } \\
2452-2444(\mathrm{~m})\end{array}$ & & $\begin{array}{c}\text { Perforation } \\
\text { range } \\
2552-2545 \\
(\mathrm{~m})\end{array}$ & $\begin{array}{c}\text { Perforation range } \\
2516 \\
-2504(\mathrm{~m})\end{array}$ & & \\
\hline $\mathrm{CH}_{4}$ & 92,76 & 89,14 & 90,95 & 92,39 & 90,99 & 91,69 & 92,60 \\
\hline $\mathrm{C}_{2} \mathrm{H}_{6}$ & 2,56 & 4,50 & 3,53 & 2,46 & 4,57 & 3,52 & 3,44 \\
\hline $\mathrm{C}_{3} \mathrm{H}_{8}$ & 1,89 & 2,21 & 2,05 & 1,92 & 0,65 & 1,29 & 0,72 \\
\hline iso- $\mathrm{C}_{4} \mathrm{H}_{10}$ & 0,45 & 0,51 & 0,48 & 0,32 & 0,37 & 0,35 & 0,20 \\
\hline $\mathrm{n}-\mathrm{C}_{4} \mathrm{H}_{10}$ & 0,44 & 0,54 & 0,49 & 0,27 & 0,41 & 0,34 & 0,25 \\
\hline $\mathrm{C}_{5} \mathrm{H}_{12+\text { higher }}$ & 0,90 & 1,08 & 0,99 & 1,20 & 0,80 & 1,0 & 0,66 \\
\hline $\mathrm{N}_{2}$ & 0,48 & 1,24 & 0,86 & 0,71 & 1,58 & 1,15 & 1,34 \\
\hline $\mathrm{H}_{2} \mathrm{~S}$ & - & - & - & - & - & - & - \\
\hline $\mathrm{CO}_{2}$ & 0,52 & 0,78 & 0,65 & 0,72 & 0,63 & 0,63 & 0,79 \\
\hline Total & 100 & 100 & 100 & 100 & 100 & 100 & 100 \\
\hline \multicolumn{8}{|c|}{ Potential condensate content, $\mathrm{g} / \mathrm{m}^{3}$} \\
\hline $\begin{array}{l}\text { - in } 1 \mathrm{~m}^{3} \text { of reservoir } \\
\text { gas }\end{array}$ & 39,24 & 48,73 & 35,68 & 57,81 & 32,32 & 45,07 & 32,11 \\
\hline - in $1 \mathrm{~m}^{3}$ of dry gas & 39,60 & 49,23 & 35,96 & 58,51 & 32,58 & 45,55 & 32,32 \\
\hline $\begin{array}{l}\text { Potential ethane } \\
\text { content, } \mathrm{g} / \mathrm{m}^{3}\end{array}$ & 32,02 & 56,28 & 44,15 & 30,77 & 57,16 & 44,03 & 43,03 \\
\hline \multicolumn{8}{|c|}{ Critical parameters: } \\
\hline$-P_{\text {cr. }}, \mathrm{kgf} / \mathrm{cm}^{2}$ & 46,80 & 46,84 & 46,81 & 46,74 & 46,81 & 46,77 & 46,90 \\
\hline$-\mathrm{T}_{\text {cr. }}, \mathrm{K}$ & 202,32 & 205,09 & 203,8 & 203,08 & 201,14 & 202,40 & 199,34 \\
\hline $\begin{array}{l}\text { Gas density at } 20^{\circ} \mathrm{C}, \\
760 \mathrm{~mm} \mathrm{Hg}, \mathrm{kg} / \mathrm{m}^{3}\end{array}$ & 0,763 & 0,782 & 0,773 & 0,777 & 0,760 & 0,764 & 0,748 \\
\hline Relative gas density & 0,634 & 0,649 & 0,642 & 0,645 & 0,631 & 0,634 & 0,621 \\
\hline
\end{tabular}

Table 3. Physical and chemical characteristics of the gases of North Shurtan field

\begin{tabular}{|l|c|c|}
\hline & Free gas & Oil dissolved gas \\
\hline Density (by air) & 0,652 & 0,706 \\
\hline Content, in volume \%: & \multicolumn{2}{|c|}{} \\
\hline methane & 87,8 & 6,34 \\
\hline ethane & 4,98 & 3,84 \\
\hline propane & 2,48 & 1,25 \\
\hline isobutane & 0,56 & 1,79 \\
\hline normal butane & 0,64 & 0,7 \\
\hline isopentane & 0,18 & 0,45 \\
\hline normalpentane & 0,09 & 0,05 \\
\hline hydrogensulfide & 0,15 & 1,58 \\
\hline carbondioxide & 1,68 & 2,68 \\
\hline nitrogen & 1,44 & \\
\hline Calorificvalue, $\mathrm{kcal} / \mathrm{nm}^{3}$ & 8712 & \\
\hline
\end{tabular}

The pressure reduction of the transported natural gas is carried out in two stages. At the first stage - at gas distribution stations - gas pressure decreases from the pressure in the main gas pipeline from 5.5 $\mathrm{MPa}$ to 1.2 $\mathrm{MPa}$, at the second stage - at gas control points - from 1.2 to $0.15 \mathrm{MPa}$. In the process of expansion of highpressure natural gas in EGU, its pressure decreases to 1.2 $\mathrm{MPa}$ and temperature - to $10^{\circ} \mathrm{C}$. [6-9].

Based on the above, a statement of the problem of optimization was formed and presented in the following form: annual electricity generation

$$
\max E=f(P, G),
$$

where $P €\left[P_{1}, P_{2}\right] ; G \in\left[G_{1}, G_{2}\right]$.

Limitations

$T_{2}=0 ; \quad T_{1} \leq T_{\text {мах }} ; \quad Q_{T O} \leq Q_{\text {мах }} ; \quad N_{Э Г} \leq N_{\text {мах }}$. (2)

Due to the fact that expansion with the help of an expander leads to a more significant decrease in the gas temperature, for economic comparison the fuel gas consumption for a fire heater with a characteristic thermal $\mathrm{h}=40 \%$ was taken into account in the calculations. 
The calculations were performed using the GasCondOil thermodynamic calculation program [10], and the results are presented in Table 4.

Table 4. Comparison of natural gas expansion parameters on throttle and expander

\begin{tabular}{|c|c|c|c|c|}
\hline \multirow[t]{2}{*}{ Type } & \multirow[t]{2}{*}{ Parameter } & \multicolumn{3}{|c|}{$\begin{array}{l}\text { Outlet gas pressure, } \\
\qquad \mathrm{MPa}\end{array}$} \\
\hline & & 0,3 & 0,6 & 1,2 \\
\hline \multirow{3}{*}{ Throttle } & Transition gas temperature after throttle, ${ }^{\circ} \mathrm{C}$ & $-11,8$ & $-9,6$ & $-6,2$ \\
\hline & Heat quantity to heat transit gas after throttle $\left(\mathrm{t}_{\mathrm{k}}=10^{\circ} \mathrm{C}\right), \mathrm{kJ} /$ hour $(\mathrm{kW})$ & $\begin{array}{l}693000 \\
(190,5)\end{array}$ & $\begin{array}{r}639700 \\
(170,7)\end{array}$ & $\begin{array}{l}532500 \\
(140,0)\end{array}$ \\
\hline & $\begin{array}{l}\text { Consumption of fuel gas for heating transit gas after throttle }(\mathrm{h}=40 \%) \text {, } \\
\mathrm{nm}^{3}\end{array}$ & 50 & 46 & 40 \\
\hline \multirow{4}{*}{$\begin{array}{l}\text { Expander } \\
\text { (heating } \\
\text { after) }\end{array}$} & Transit gas temperature after expander, ${ }^{\circ} \mathrm{C}$ & $-102,4$ & $-81,2$ & $-50,3$ \\
\hline & Expander capacity, kW & 830 & 650 & 450 \\
\hline & Heat quantity to heat transit gas after expander $\left(\mathrm{t}_{\mathrm{k}}=10^{\circ} \mathrm{C}\right), \mathrm{kJ} /$ hour $(\mathrm{kW})$ & $\begin{array}{l}700790 \\
(1025)\end{array}$ & $\begin{array}{l}3027500 \\
(835)\end{array}$ & $\begin{array}{l}2186600 \\
(600)\end{array}$ \\
\hline & $\begin{array}{l}\text { Consumption of fuel gas for heating transit gas after expander } \\
(\mathrm{h}=40 \%), \mathrm{nm}^{3}\end{array}$ & 270 & 220 & 160 \\
\hline \multirow{4}{*}{$\begin{array}{l}\text { Expander } \\
\text { (heating } \\
\text { before) }\end{array}$} & Heat quantity to heat transit gas before expander $\left(t_{k}=10^{\circ} \mathrm{C}\right), \mathrm{kJ} /$ hour $(\mathrm{kW})$ & $\begin{array}{l}5948900 \\
(1650)\end{array}$ & $\begin{array}{l}4341900 \\
(1200)\end{array}$ & $\begin{array}{l}2795800 \\
(770)\end{array}$ \\
\hline & Expander capacity, kW & 1400 & 1000 & 600 \\
\hline & $\begin{array}{l}\text { Consumption of fuel gas for heating transit gas before expander } \\
(\mathrm{h}=40 \%), \mathrm{nm}^{3}\end{array}$ & 440 & 320 & 200 \\
\hline & Transit gas temperature before expander, ${ }^{\circ} \mathrm{C}$ & 160,5 & 120,2 & 83,5 \\
\hline
\end{tabular}

Thus, the calculations and operating experience of expander-generator sets confirm the value of relative power generation in the amount of $40 \ldots 60 \mathrm{~kW} /$ thousand nm3 and make it possible not only to introduce secondary energy resources into the economy and provide the generation of electricity, but also to reduce the level of harmful emissions in comparison with traditional technologies.

\section{References}

1.URL: http: //old.uztransgaz.uz/ru/content/osnovnye-pokazateli

2. V.Gurov, EES, 4, 22-24 (2006).

3. O.Malkhanov, RSE, 1, 39-41 (2008).

4. E.Marchenko, O.Malkhanov, RSE, 6, 38-42 (2009).

5. H.Fasold, GEG, 6, 261-269 (1995).

6. A.Gafurov, BKSEU, 1, 28-36 (2014)

7. I.Kharisov, STSSPbSPU, 1, 119-123 (2012).

8. B.Agababov, ESWT, 1, 71-73 (2011).

9. O.Sorokin, IPE, 6, 36-41 (2005).

10. // URL: http://gascondoil.com/GazCondOil 